\title{
The dynamical disconnection of sunspots from their magnetic roots
}

\author{
M. Schüssler ${ }^{1}$ and M. Rempel ${ }^{2}$ \\ 1 Max-Planck-Institut für Sonnensystemforschung, Max-Planck-Str. 2, 37191 Katlenburg-Lindau, Germany \\ e-mail: msch@mps.mpg.de \\ ${ }^{2}$ High Altitude Observatory, NCAR, PO Box 3000, Boulder, Colorado 80307, USA
}

Received 1 March 2005 / Accepted 13 June 2005

\begin{abstract}
After a dynamically active emergence phase, magnetic flux at the solar surface soon ceases to show strong signs of the subsurface dynamics of its parent magnetic structure. This indicates that some kind of disconnection of the emerged flux from its roots in the deep convection zone should take place. We propose a mechanism for the dynamical disconnection of the surface flux based upon the buoyant upflow of plasma along the field lines. Such flows arise in the upper part of a rising flux loop during the final phases of its buoyant ascent towards the surface. The combination of the pressure buildup by the upflow and the cooling of the upper layers of an emerged flux tube by radiative losses at the surface lead to a progressive weakening of the magnetic field in several Mm depth. When the field strength has become sufficiently low, convective motions and the fluting instability disrupt the flux tube into thin, passively advected flux fragments, thus providing a dynamical disconnection of the emerged part from its roots. We substantiate this scenario by considering the quasi-static evolution of a sunspot model under the effects of radiative cooling, convective energy transport, and pressure buildup by a prescribed inflow at the bottom of the model. For inflow speeds in the range shown by simulations of thin flux tubes, we find that the disconnection takes place in a depth between 2 and $6 \mathrm{Mm}$ for disconnection times up to 3 days.
\end{abstract}

Key words. magnetohydrodynamics (MHD) - Sun: activity - Sun: magnetic fields - Sun: sunspots

\section{Introduction}

Until the advent of local helioseismology, very little was known about the subsurface structure of sunspots and active regions. Differences in the measured rotation rates of the nonmagnetic plasma and the magnetic "tracers" were taken as evidence that the latter are somehow "anchored" in deeper layers, but no consensus could be reached as to where and how this anchoring should precisely take place (e.g., D'Silva \& Howard 1994; Javaraiah \& Gokhale 1997; Beck 2000; Schüssler 1984, 1987). The recent results from local helioseismology indicate significant changes of the thermodynamic and/or magnetic properties as well as of the flow field at a depth of less than $10 \mathrm{Mm}$ below sunspots (Kosovichev et al. 2000; Zhao et al. 2001; Couvidat et al. 2004) and active regions (Basu et al. 2004; Hindman et al. 2004). These findings are consistent with the model of a sunspot as a shallow object, which breaks apart into a large number of flux tubes not far below the surface (Parker 1979; Spruit 1981; Choudhuri 1992), rather than that of a monolithic plug of magnetic flux extending deep into the convection zone (see also the detailed discussion of sunspot models by Thomas \& Weiss 1992).

From the theoretical side, analytical studies and numerical simulations of magnetic flux tubes have provided a consistent picture of the formation of active regions and sunspot groups, comprising the storage and amplification of magnetic flux near the bottom of the convection zone as well as the formation of unstable loops and their rise through most of the convection zone. Given a strongly super-equipartition initial field of the order of $10^{5} \mathrm{G}$ (10 Tesla) at the bottom of the convection zone, large-scale properties of newly-emerged active regions and sunspot groups, like low emergence latitudes, systematic tilt angles (Joy's law), and asymmetric proper motions of the two polarities, can be quantitatively explained (see reviews by Moreno-Insertis 1997; Fisher et al. 2000; Fan 2004, and further references therein).

Numerical studies following the evolution of an unstable flux loop from its origin at the bottom of the convection zone only reach until about $10 \mathrm{Mm}$ from the surface, where the thinflux-tube approximation breaks down. Realistic simulations of the subsequent phases are still too demanding in view of the computing power available today. Therefore, the actual emergence of flux in the photosphere and the evolution thereafter has not been covered by such simulations (except for rather idealized situations aiming at describing the effects on the coronal magnetic structure, e.g., Fan \& Gibson 2004; Magara 2004; Archontis et al. 2004). On the other hand, a wealth of observational evidence indicates a remarkable change in the dynamical properties of sunspots and active regions from "active" 
to "passive" evolution shortly after their emergence (Schüssler 1987; Schrijver \& Title 1999). The emerging flux initially displays the clear signature of a rising, fragmented flux tube and evolves according to its internal large-scale dynamics (e.g., McIntosh 1981; Strous et al. 1996). However, the expansion of the bipolar region and the proper motion of the sunspots with respect to the surrounding plasma decays within a few days after emergence and larger magnetic structures start to fragment into small-scale flux bundles, which are largely dominated by the local near-surface flows (granulation, supergranulation, differential rotation, meridional circulation). The magnetic flux is then passively transported by these velocity fields and becomes dispersed over wide areas ${ }^{1}$, a process well represented by the so-called surface transport models (e.g., Wang et al. 1989; van Ballegooijen et al. 1998; Schrijver 2001; Baumann et al. 2004). This behavior is in striking contrast to what would be expected if the emerged flux would follow the evolution of its magnetic roots at the bottom of the convection zone:

Longitudinal drift: the size range of bipolar magnetic regions corresponds to azimuthal wavenumbers between $m=10$ and 60, while the magnetic instabilities leading to loop formation favor values of $m=1$ or 2 (Spruit \& van Ballegooijen 1982; Schüssler et al. 1994; Ferriz-Mas \& Schüssler 1995). Consequently, the two polarities of an active region should move apart in longitudinal direction much further than actually observed. Moreover, there is no static equilibrium of the subsurface vertical "legs" of an emerged bipolar region that is connected to an azimuthal field of $10^{5} \mathrm{G}$ at the bottom of the convection zone (van Ballegooijen 1982). The horizontal component of the magnetic tension force resulting from the bends of the flux tube where it turns horizontal sustains the drift of the two legs (and their associated poles at the surface) in opposite azimuthal directions. Thin-tube simulations in fact clearly show that the two poles drift apart unrestrained and even move around the whole circumference of the Sun within a few months. Clearly, no such systematic motion is observed.

Poleward drift: the emergence of a loop cuts off the azimuthal flow neccessary for mechanical equilibrium of the submerged field (Moreno-Insertis et al. 1992). As a result, the magnetic structure starts to drift poleward in response to the unbalanced latitudinal component of the magnetic tension force. No such systematic poleward drift is observed in bipolar regions or sunspot groups.

Tilt angle: the tilt angle of sunspot groups with respect to the East-West direction arises through the action of the Coriolis force on a rising and horizontally expanding flux loop (e.g., D'Silva \& Choudhuri 1993). After emergence, the expansion stops and the flux tube should relax back to its original EastWest orientation during the lifetime of a large bipolar region

\footnotetext{
1 When the average flux density exceeds a limit of about $100 \mathrm{G}$, the relatively stable "plage state" (Schrijver 1987) is maintained as an intermediate stage: the convective pattern is severly disturbed by the magnetic field and supergranulation is largely absent, so that the dispersal of the flux is temporarily diminished. Sunspots actively shape the surrounding flow structure by developing the outward moat flow, which probably temporarily suppresses the flux transport by supergranulation and meridional flow.
}

(Fan et al. 1994). No such relaxation is observed (Tóth \& Gerlei 2004).

The observed change of the dynamics of magnetic structures from "active" to "passive" and the points listed above clearly indicate that bipolar magnetic regions somehow become disconnected from their deeper roots within a few days after emergence. Moreover, since the expected motions of sunspots would otherwise be quite marked, the complete absence of such behaviour indicates that the disconnection process must reliably work, virtually without exception. This represents an important constraint.

To our knowledge, two mechanisms for the disconnection of emerged flux have been proposed so far. Schrijver \& Title (1999) have suggested subsurface reconnection of the two opposite polarities leading to disconnected U-loops in the upper layers. It does not seem obvious how this mechanism can achieve the required perfect reliability in view of the strong tendency of the poles to move apart in the longitudinal direction (see Sect. 2). Earlier, Fan et al. (1994) had given arguments for the necessity of disconnection and proposed a mechanism for the "dynamical disconnection" of emerged flux by a local loss of lateral total pressure balance following the establishment of an isentropic hydrostatic equilibrium along magnetic field lines. As discussed in Sect. 2, the process sketched by Fan et al. (1994) does not seem to operate sufficiently rapidly to provide disconnection within the first few days after emergence. Here we propose a variation of the dynamical disconnection scenario, which is based upon the strong, buoyancydriven upflow associated with the final phase of the rise of a flux loop through the convection zone. We discuss the various scenarios for disconnection in Sect. 2 and provide an illustrative quantitative elaboration of our model in Sect. 3. The results are discussed and put into a broader perspective in Sect. 4.

\section{Scenarios for the disconnection of emerged flux}

Schrijver \& Title (1999) have suggested that active regions literally become disconnected from their roots by a subsurface reconnection between the opposite polarities in their (fragmented) legs; this would create shallow O- or U-loops, which then could float freely with the near-surface velocity fields. The problem with this concept is that, as explained above, the two legs of a bipolar region tend to rapidly move apart from each other owing to the geometry of the rising loop and as a result of the unbalanced tension force in the very deep part. Thus is would require a rather organized and converging flow to bring the opposite fluxes together to reconnect. Furthermore, the more separated the two legs already are, the more difficult is it to bring the opposite-polarity flux together. Consequently, once reconnection has failed to occur in time for whatever reason and the two poles continue to drift further apart, they cannot be disconnected any more and separate indefinitely. To our knowledge, not a single example of such a behaviour of a larger active region has been reported by observers.

Fan et al. (1994) have suggested that the establishment of hydrostatic equilibrium along a flux tube could lead to a dynamical disconnection of the emerged part from its deeper magnetic roots, which does not explicitely require a change of 
the magnetic topology (i.e., reconnection): as the subsurface part of the emerged flux tube approaches hydrostatic equilibrium, it eventually loses lateral pressure equilibrium at a certain height. This leads to a region of weak, passive magnetic field that effectively decouples the parts of the flux tube above and below this height.

This mechanism is closely related to the "explosion" of magnetic flux tubes discussed by Moreno-Insertis et al. (1995): a slowly rising flux loop that maintains approximate hydrostatic equilibrium along the field lines experiences a sudden catastrophic weakening of the field strength at its apex when it reaches a critical height. This explosion height depends on the field strength in the part of the flux tube that remains at the bottom of the convection zone. The plasma is nearly isentropic within the adiabatically rising flux loop whereas the entropy decreases with height in the surrounding superadiabatically stratified convection zone. As a consequence of the higher entropy within the magnetic flux tube, the internal pressure decreases more slowly with height than the external pressure and thus eventually both become equal at a certain height above the bottom of the convection zone (Schüssler \& Rempel 2002). When the loop apex approaches this "explosion height", it expands drastically since pressure balance forces the magnetic field to become very small, so that it is no longer dynamically relevant (Rempel \& Schüssler 2001). From mixing-length models of the solar convection zone one finds that a hydrostatic flux tube with a field strength of $\mathrm{O}\left(10^{5}\right) \mathrm{G}$ at the base of the convection zone should explode at a depth of less than $10 \mathrm{Mm}$ below the surface. On the other hand, dynamical simulations show that rising flux loops with such initial field strengths actually traverse this height range without exploding because a flow along the field lines keeps them sufficiently far away from hydrostatic equilibrium (e.g., Caligari et al. 1995).

The problem with the scenario of Fan et al. (1994) is the timescale for the establishment of the "global" hydrostatic equilibrium required for the disconnection. The associated time scale if of the order of the Alfvén travel time along the circumference of the flux tube: taking an Alfvén speed of $1 \mathrm{~km} \mathrm{~s}^{-1}$ (corresponding to $10^{5} \mathrm{G}$ at the bottom of the convection zone), this corresponds to about a month, much too long to avoid an unrealistic evolution of the emerged part.

However, a more detailed consideration of the development in the upper parts of an emerged flux tube reveals a much faster and more local route towards dynamical disconnection, avoiding the requirement of a global hydrostatic equilibrium. Consider the following situation. A rising flux loop has emerged at the surface to form a bipolar magnetic region. The subsurface part of the loop has not exploded at its formal explosion height because the strongly buoyant and rapidly rising plasma in the loop maintains a super-hydrostatic gradient of the gas pressure (i.e., a steeper upward decline of the pressure than in the hydrostatic case) connected with an accelerated upflow (Caligari et al. 1995). After emergence, the near-surface parts of the sunspots, pores, and other flux concentrations are rapidly cooled by radiative losses, which gives rise to an inward propagating cooling front accompanied by a local downflow. This leads to a decrease of the gas pressure and a concomitant intensification of the magnetic field in the upper layers of the flux tube, while the upflow from below and the downflow from above increase the gas pressure below the first few $\mathrm{Mm}$ depth. After a few days, the gas pressure has increased sufficiently to approach the ambient pressure somewhere between 2 and $10 \mathrm{Mm}$ depth. A thin flux tube (requiring lateral balance of total pressure) would then lose its equilibrium and explode. A more general magnetostatic equilibrium could possibly be maintained for some time but, in any case, the magnetic field becomes strongly weakened. Once the field strength falls below the equipartition value with respect to the external convective motions, the field becomes largely passive in that height range and progressively fragments, so that the upper, magnetostatic part becomes dynamically disconnected from its roots.

\section{A simple model for dynamical disconnection}

In order to evaluate the explanatory potential of the scenario sketched above and to provide an illustrative example, we study the quasi-static evolution of a sunspot model very similar to that of Deinzer (1965).

\subsection{Model description}

\section{Evolution}

To realistically follow the sequence of events from flux tube emergence to disconnection would require a fully dynamic realistic numerical simulation, which is beyond the scope of this paper. We restrict ourselves to sequences of quasi-static models of a sunspot and its underlying flux tube, which evolve in reaction to surface cooling and to the increasing pressure resulting from an inflow through the lower boundary of the model. Such an approach is justified as long as the upflow velocity is significantly smaller than the Alfvén speed, so that the magnetic structure remains almost unaffected by the dynamical pressure of the flow. Starting from an isentropic initial state, we proceed in constant intervals of time and, for each time step, determine the entropy change due to radiative and convective energy transport. Next we integrate the gas pressure, taking into account the growing base pressure owing to the assumed upflow, apply the corresponding adiabatic correction to the temperature, and calculate the modified magnetostatic equilibrium. Then we move to the next timestep. In the following subsections we explain the various ingredients of the model in more detail.

\section{Magnetostatic equilibrium}

We basically follow the approach of Deinzer (1965) and consider a similarity solution (Schlüter \& Temesváry 1958) for an axisymmetric magnetostatic configuration with hydrostatic equilibrium along the field lines and a mixing-length model of the convection zone (Kiefer et al. 2000) representing the (fixed) external stratification.

The similarity model assumes an axisymmetric magnetic structure with a self-similar profile of the magnetic field as a function of the radial coordinate, $r$, in cylindric coordinates. 
The two components of the (untwisted) magnetic field are written in the form

$B_{z}(r, z)=f(\zeta) B_{0}(z)$

$B_{r}(r, z)=-\frac{r}{2} f(\zeta) \frac{\partial B_{0}(z)}{\partial z}$

where $B_{0}(z)$ is the magnetic field along the symmetry axis and $\zeta=r \sqrt{B_{0}(z)}$. The function $f(\zeta)$, which describes the radial profile of the vertical field component, can be freely chosen. We follow the usual practice and use a Gaussian: $f(\zeta)=\exp \left(-\zeta^{2}\right)$. Inserting the ansatz given by Eqs. (1) and (2) into the equations for magnetostatic equilibrium,

$0=-\frac{\partial p}{\partial r}+\frac{B_{z}}{4 \pi}\left(\frac{\partial B_{r}}{\partial z}-\frac{\partial B_{z}}{\partial r}\right)$

$0=-\frac{\partial p}{\partial z}-\frac{B_{r}}{4 \pi}\left(\frac{\partial B_{r}}{\partial z}-\frac{\partial B_{z}}{\partial r}\right)-\rho g$,

and integrating the first of these over $r$ (from 0 to $\infty$ ) for constant $z$, yields the ordinary differential equation

$\frac{\Phi}{2 \pi} y \frac{\mathrm{d}^{2} y}{\mathrm{~d} z^{2}}=y^{4}-8 \pi\left(p_{\mathrm{e}}-p_{\mathrm{i}}\right)$

where $y=\sqrt{B_{0}(z)}, \Phi$ is the total magnetic flux, $p_{\mathrm{e}}(z)=p(\infty, z)$ is the (fixed) external pressure, and $p_{\mathrm{i}}=p(0, z)$ is the pressure on the axis. The latter is simply given by hydrostatic equilibrium along the axis, viz.

$\frac{\mathrm{d} p_{\mathrm{i}}}{\mathrm{d} z}=\varrho_{\mathrm{i}} g$

where $\varrho_{\mathrm{i}}(z)$ is the density profile along the axis and $g(z)$ is the (depth-dependent) gravitational acceleration. Consequently, the self-similarity assumption reduces the determination of the magnetohydrostatic force balance to solving two ordinary differential equations, namely hydrostatic equilibrium along the central field line of the axisymmetric configuration and an equation relating the field strength on the axis to the difference between the gas pressure on the axis and the (external) pressure far away from the axis. Note that the thin flux tube approximation with $B^{2}=y^{4}=8 \pi\left(p_{\mathrm{e}}-p_{\mathrm{i}}\right)$ is recovered in the limit $\Phi \rightarrow 0$. The left-hand side of Eq. (5) represents the contribution of magnetic stress integrated from the axis of the field configuration to infinity, which is not considered in the thin flux tube limit. A detailed derivation of Eq. (5) can be found in Schlüter \& Temesváry (1958).

We solve Eq. (5) through an iterative relaxation procedure,

$y_{n+1}=y_{n}+\frac{\varepsilon}{y_{n}^{3}}\left[\frac{\Phi}{2 \pi} y_{n} \frac{\mathrm{d}^{2} y_{n+1}}{\mathrm{~d} z^{2}}-y_{n}^{3} y_{n+1}+8 \pi\left(p_{\mathrm{e}}-p_{\mathrm{i}}\right)\right]$,

where $y_{n}(z)$ is the $n$th iteration of the solution. This procedure avoids numerical stiffness problems introduced by the term $y^{4}$ and allows us to specify boundary conditions for $y$ on both sides of the computational domain. The prefactor $y_{n}^{-3}$ has been introduced in order to increase the convergence and stability of the algorithm, which we optimize by the choice of the free parameter $\varepsilon$.

\section{Energy transport}

We consider vertical radiative transfer in the grey diffusion approximation. Since the energy flux of a sunspot umbra cannot be provided by radiation alone, a reduced level of convective energy transport is required in addition (Deinzer 1965). The phenomenon of umbral dots is often taken as a manifestation of small-scale convection in the strong umbral magnetic field. In the absence of a better theoretical model, we treat the magneto-convective energy transport using a mixing-length approach, taking into account the inhibiting effect of the magnetic field through a reduction of the mixing-length parameter. Horizontal radiative energy exchange can be neglected in the case of sunspots, which are much larger than the photon mean free path of the order of $100 \mathrm{~km}$ at optical depth unity. The time evolution of $T_{\mathrm{i}}$ then follows from

$\varrho_{\mathrm{i}} c_{\mathrm{p}} \frac{\partial T_{\mathrm{i}}}{\partial t}=-\frac{\partial}{\partial z}\left(F_{\text {rad }}+F_{\text {conv }}\right)$

where the radiative energy flux is given by

$F_{\mathrm{rad}}=-\frac{16 \sigma T_{\mathrm{i}}^{3}}{3 \kappa_{R} \varrho_{\mathrm{i}}} \frac{\partial T_{\mathrm{i}}}{\partial z}$.

The Rosseland mean opacity, $\kappa_{R}\left(\varrho_{\mathrm{i}}, T_{\mathrm{i}}\right)$, is interpolated from a table. Since the value of $\kappa_{R}$ significantly drops in the surface layers, the radiative heat conductivity reaches large values, so that we use a semi-implicit treatment of the radiative energy flux in order to avoid a too severe time step constraint.

The convective energy flux along the flux tube is determined following Spruit (1974),

$F_{\text {conv }}=-b \sqrt{a}\left(\frac{\mathcal{R}}{\mu}\right)^{1 / 2}\left(\frac{l}{H_{\mathrm{p}}}\right)^{1 / 2} \varrho_{\mathrm{i}} c_{\mathrm{p}} T_{\mathrm{i}}^{3 / 2}\left(\nabla-\nabla^{\prime}\right)^{3 / 2}$

where $\nabla$ is the logarithmic temperature gradient with respect to the logarithm of pressure, $\mu$ is the average molecular weight, $\mathcal{R}$ the gas constant, and $l / H_{\mathrm{p}}$ the (constant) mixing length parameter as fraction of the local pressure scale height. The logarithmic temperature gradient gradient $\nabla^{\prime}$ reflects radiative energy exchange of the convective parcels and follows from

$\nabla^{\prime}=\nabla_{\mathrm{ad}}-2 u^{2}+2 u\left(\nabla-\nabla_{\mathrm{ad}}+u^{2}\right)^{1 / 2}$,

with

$u=\frac{1}{f \sqrt{a}}\left(\frac{l}{H_{\mathrm{p}}}\right)^{-2} \frac{12 \sigma T_{\mathrm{i}}^{3}}{c_{\mathrm{p}} \varrho_{\mathrm{i}} \kappa_{R} H_{\mathrm{p}}^{2}}\left(\frac{H_{\mathrm{p}}}{g}\right)^{1 / 2}$.

Here $\nabla_{\text {ad }}$ denotes the adiabatic logarithmic temperature gradient and $\sigma$ is Stefan's radiation constant. The values of the geometric parameters used are $a=1 / 8, b=1 / 2$, and $f=3 / 2$.

In the case of a very strong systematic vertical flow with a time scale comparable to the turnover time of the convective motions, the mixing-length approach may be invalidated and, in addition, the advective entropy transport by the largescale flow may become relevant. In our simulations, such a situation occurs only during the initial cooling phase when a rather strong transient downflow develops in the first 1-2 Mm depth (see the subsection on the implied vertical velocity below). For the later evolution of the configuration, the mixing length model appears to be adequate for the purposes of an illustrative model. 


\section{Equation of state}

We include the partial ionization of $\mathrm{H}, \mathrm{He}$, and $\mathrm{He}^{+}$. The values of density, heat capacity, and adiabatic temperature gradient as functions of pressure and temperature are determined in the course of the calculation by interpolation in a pre-compiled table.

\section{Boundary conditions}

For the temperature, we have to specify boundary conditions at both ends of the integration domain. At the upper boundary ( $z=0$, taken to be the level of Rosseland optical depth unity of the external stratification) we fix the temperature to a value (typically $3500 \mathrm{~K}$ ) that is lower than the temperature expected at the level $\tau=2 / 3$ in the sunspot. At the bottom, we keep the temperature gradient adiabatic.

For solving Eqs. (6) and (5) we specify a boundary condition for $p_{\mathrm{i}}$ at the bottom of the integration domain and values for $y$ at the bottom and at the top, respectively. The magnetic field strength is fixed at the upper boundary (typically at a value of $2000 \mathrm{G})$, while the thin flux tube relation, $B=\sqrt{8 \pi\left(p_{\mathrm{e}}-p_{\mathrm{i}}\right)}$, is assumed to hold at the bottom.

We integrate the hydrostatic balance upward from the lower boundary, specifying the value of the base pressure for each time step according to the influx of mass due to the assumed upflow. The effect of lateral expansion or contraction of the configuration is taken into account in the calculation of the base pressure. We can alternatively specify a constant upflow velocity, $v_{0}$, or a constant total mass flux through the lower boundary of our integration domain.

In the thin flux tube approximation, the total mass within a flux tube containing the (constant) magnetic flux $\Phi$ is given by $m=\Phi \int_{0}^{z_{0}} \frac{\varrho_{\mathrm{i}}}{B} \mathrm{~d} z$

where $z_{0}$ represents the lower boundary of the integration domain. The base pressure, $p_{\mathrm{i}}\left(z_{0}\right)$, is adjusted each time step such that the change in total mass, $\Delta m$, reflects the inflow of material across the boundary,

$\Delta m=A\left(z_{0}\right) \varrho_{\mathrm{i}}\left(z_{0}\right) v_{0} \Delta t=\Phi \frac{\underline{\varrho}_{\mathrm{i}}\left(z_{0}\right)}{B\left(z_{0}\right)} v_{0} \Delta t$,

where $A(z)=\Phi / B(z)$ is the cross-sectional area of the flux tube. These relations also hold more generally for the self-similar solutions considered here, since the profile of the magnetic field in radius is the same at each depth, $z$, except for a scaling factor. Therefore, we can define an "effective" cross section of the configuration at each height on the basis of the axial field as $A_{\text {eff }}(z)=\Phi / B(z)$, where $A_{\text {eff }}$ depends on the radial field profile considered. $A_{\mathrm{eff}}$ is in general different from the geometric diameter of the flux configuration (for instance, in the case of a Gaussian profile it formally has infinite extent). However, since the total mass of the flux tube and the mass flux across the lower boundary scale in the same way with the cross-section, the difference between $A_{\mathrm{eff}}$ and the geometric cross-section has no influence on the solution.

A change of the pressure at the base, $p_{\mathrm{i}}\left(z_{0}\right)$, alters the mass within the flux tube through a change of $\varrho_{\mathrm{i}}$ and $B$ (the latter is the dominant contribution since $\beta=8 \pi p_{i} / B^{2} \gg 1$ except for the uppermost layers), which can be written formally as $m=m\left[p_{\mathrm{i}}\left(z_{0}\right)\right]$. The adjustment of the base pressure $\Delta p_{\mathrm{i}}\left(z_{0}\right)$ has to be determined such that

$m\left[p_{\mathrm{i}}\left(z_{0}\right)+\Delta p_{\mathrm{i}}\left(z_{0}\right)\right]=m\left[p_{\mathrm{i}}\left(z_{0}\right)\right]+\Phi \frac{\varrho_{\mathrm{i}}\left(z_{0}\right)}{B\left(z_{0}\right)} v_{0} \Delta t$

holds. Since the relation between $\Delta p_{\mathrm{i}}\left(z_{0}\right)$ and $\Delta m$ is non-linear (mainly through Eq. (5)), we use a Newton iteration to determine the adjustment of the base pressure such that Eq. (15) is satisfied. Note that each iteration step requires a full integration of the Eqs. (6) and (5).

For reasons of consistency with the self-similar solution it is required that the flow velocity has no variation over the cross section of the flux tube. This ensures that the magnetic field profiles remain self similar at all times.

\section{Implied vertical velocity}

The inflow at the bottom, the radiative and convective cooling, and the resulting changes of the magnetostatic balance lead to a net change as well as to a redistribution of mass within the magnetic structure. In our quasi-static model, we can determine the implied vertical velocity on the axis by considering the equation of continuity:

$\frac{\partial}{\partial t} \frac{\underline{\varrho}_{\mathrm{i}}}{B}+\frac{\partial}{\partial z}\left(v \frac{\varrho_{\mathrm{i}}}{B}\right)=0$

Starting with $v=0$ at the upper boundary, integration along the tube axis leads to:

$v(z)=-\frac{B(z)}{\varrho_{\mathrm{i}}(z)} \frac{\partial}{\partial t} \int_{0}^{z} \frac{\varrho_{\mathrm{i}}}{B} \mathrm{~d} z^{\prime}$.

We determine the time derivative as a finite difference from two consecutive time steps of the hydrostatic solutions. The feedback of the dynamic pressure on the solution can be neglected as long as $\varrho_{\mathrm{i}} v^{2} / 2 \ll p_{\mathrm{e}}-p_{\mathrm{i}}$. Even for the maximum inflow velocities that we consider (of the order of $1000 \mathrm{~m} / \mathrm{s}$ ), the dynamic pressure is more than two orders of magnitude smaller than the difference between the external and internal gas pressures. In other words, the inflow velocity is at least one order of magnitude smaller than the initial Alfvén speed. In all cases that we have considered, the hydrostatic approximation continues to be valid throughout the whole simulation.

We have not considered the advection effects from the implied vertical velocity in the energy equation. In the deeper, homentropic part of the flux tube, such effects are irrelevant for the (adiabatic) temperature structure, while the downflow in the upper part could be temporarily important, but rapidly diminishes to less than $20 \mathrm{~m} \mathrm{~s}^{-1}$ after the first phase of radiative cooling. The advection of low entropy by the downflow probably enhances the intensification of the magnetic field in the upper part (in effect, this is the convective collapse mechanism). A quantitative evaluation of these effects requires a fully dynamic simulation, which is beyond the scope of this paper. 


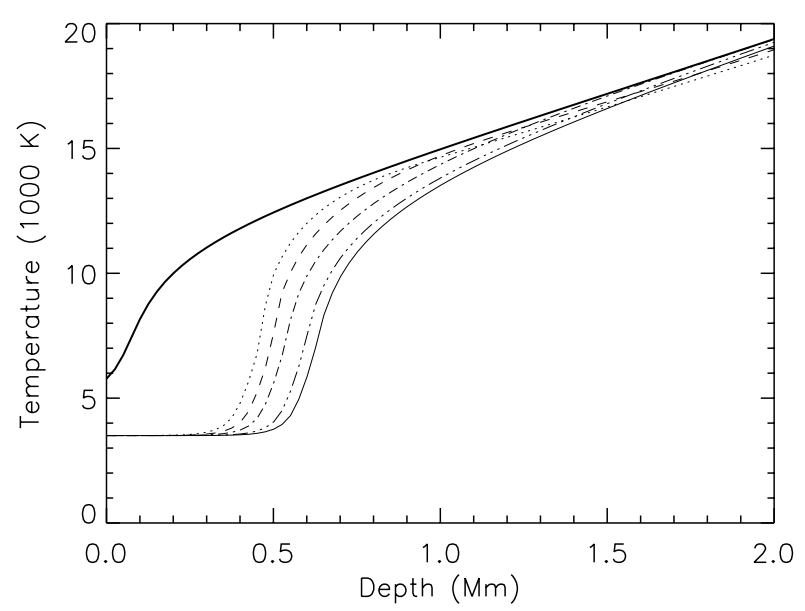

Fig. 1. Sequence of temperature profiles as a function of depth in the upper part of the model, showing the rapid inward propagation of a cooling front and the establishment of a largely stationary profile after a few hours. Zero depth corresponds to optical depth unity in the external stratification. The uppermost (thick full) curve shows the (timeindependent) external temperature. The other curves show the temperature on the sunspot axis after $0.5 \mathrm{~h}$ (dotted curve), $1 \mathrm{~h}$ (dashed curve), $4 \mathrm{~h}$ (dash-dotted curve), $15 \mathrm{~h}$ (dash-triple-dotted curve), and $30 \mathrm{~h}$ (full curve), respectively, from the onset of radiative cooling (corresponding to the emergence of the flux tube at the surface).

\section{Initial condition}

We initialize the problem with an isentropic stratification, using an entropy value equal to the entropy of external stratification at the lower boundary. Since the external stratification is strongly superadiabatic in the uppermost layers of the convection zone, the magnetic field strength at the lower boundary has to be chosen fairly large (around $100 \mathrm{kG}$ for a bottom depth of $12.5 \mathrm{Mm}$ ) in order to to avoid the loss of lateral pressure balance (i.e., an explosion) already in the initial state. This is a consequence of the magnetostatic approach chosen here. Therefore, the time evolution in the deeper layers as calculated here does not fully represent the real solar situation. On the other hand, the final state of an (nearly) exploded configuration is a fully consistent and relevant magneto-hydrostatic solution, which shows that a static sunspot is possible after dynamical disconnection.

\subsection{Numerical results}

\section{Example run}

As an illustration of a typical evolution in the framework of our quasi-static model, Figs. 1-3 show snapshots of temperature, magnetic field strength, and convective energy flux, respectively, along the axis of the flux tube. In this case we have assumed a total magnetic flux of $10^{21} \mathrm{Mx}$ and a constant mass flux at the bottom (located at $12.5 \mathrm{Mm}$ depth) corresponding to an initial inflow velocity of $700 \mathrm{~m} \mathrm{~s}^{-1}$. Simulations of thin flux tubes give rising speeds of that order at the same depth (Caligari et al. 1995). The spacing of the numerical grid is $25 \mathrm{~km}$. The temperature at the upper boundary (at optical depth unity of the external stratification) has been fixed at a value of $3500 \mathrm{~K}$ and the magnetic field strength at $2000 \mathrm{G}$. The ratio

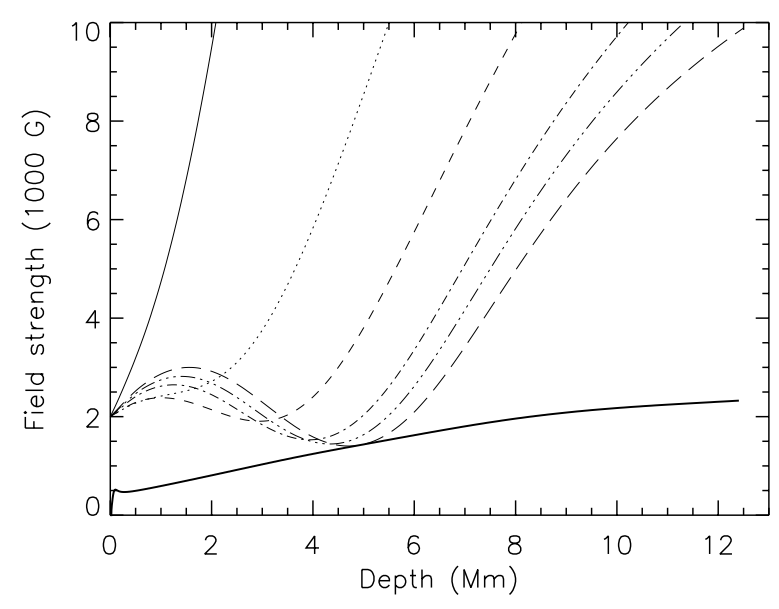

Fig. 2. Snapshots from the time evolution of the magnetic field strength along the tube axis as a function of depth for the same case as shown in Fig. 1 (note the different depth range). The curves correspond to about $1 \mathrm{~h}$ (upper full curve), $10 \mathrm{~h}$ (dotted curve), $20 \mathrm{~h}$ (short-dashed curve), $30 \mathrm{~h}$ (dash-dotted curve), $35 \mathrm{~h}$ (dash-triple-dotted curve), and $40 \mathrm{~h}$ (long-dashed curve) after the start of radiative cooling (emergence). The lower, thick full curve gives the equipartition field strength with respect to the convective velocities from the mixing length model of the external stratification (Kiefer et al. 2000). After an initial general drop, the field strength increases again down to a depth of about $4 \mathrm{Mm}$ while a local minimum of the field strength develops and moves downward until, after about $40 \mathrm{~h}$, the field strength falls below the local equipartition value at at depth of $4.7 \mathrm{Mm}$.

$\alpha=l / H_{\mathrm{p}}$ of the mixing length, $l$, to the local pressure scale height, $H_{\mathrm{p}}$, determines the total energy flux in the flux tube, which is rather well constrained by sunspot observations at $20-25 \%$ of the undisturbed solar flux. A value of $\alpha=0.3$ leads to an energy flux of about $22 \%$ of the undisturbed solar value at the top of the model. About $40 \mathrm{~h}$ after the start of radiative cooling and bottom inflow, the field strength has fallen below the equipartition value (with respect to the convective velocity in the exterior) at at depth of $4.7 \mathrm{Mm}$, implying the dynamical disconnection of the upper part from its roots. At the same time, the inflow velocity has decreased to less than $50 \mathrm{~m} \mathrm{~s}^{-1}$ owing to the strong weakening of the field and the assumed constant mass flux.

Figure 1 shows the fixed external temperature stratification (thick full line) and five profiles of the temperature in the upper part of the model at various instants of time. After the emergence of the flux tube at the photosphere (start of the calculation), radiative cooling leads to the development of a cooling front, which rapidly progresses inward. Already after about $30 \mathrm{~min}$, the level of optical depth unity in the flux tube (the Wilson depression) has reached a depth of about $375 \mathrm{~km}$ and after a few hours the inward propagation of the profile has become very slow (dash-dotted curve, after $4 \mathrm{~h}$ ). The last curve (thin full line) shows the largely stationary temperature profile about $30 \mathrm{~h}$ after the start of radiative cooling. The Wilson depression has reached a value of about $550 \mathrm{~km}$ with a temperature of about $4100 \mathrm{~K}$ at (Rosseland) optical depth unity.

For the same case, Fig. 2 shows snapshots of the magnetic field profiles along the tube axis. The full curve shows the situation after about $1 \mathrm{~h}$ with a steep inward rise of the field strength 


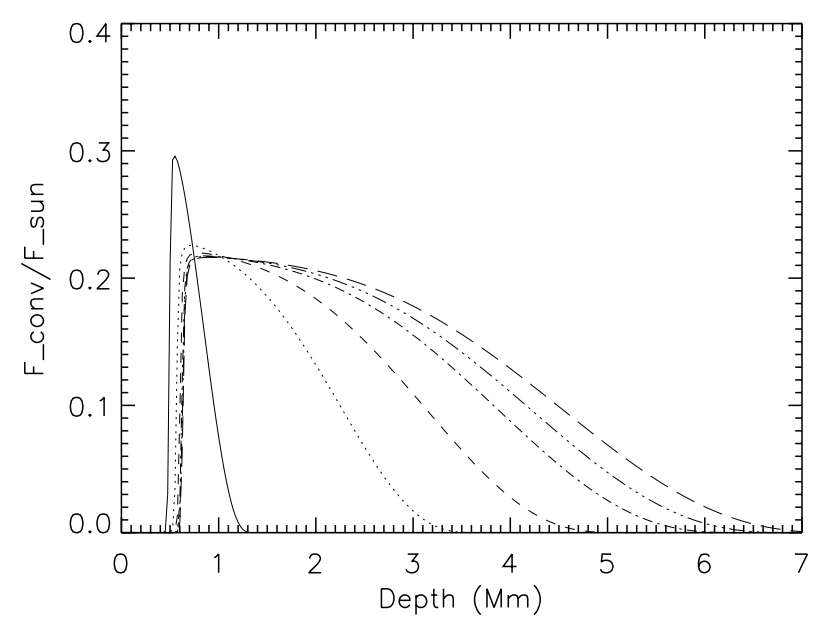

Fig. 3. Profiles of the convective energy flux density (normalized by the undisturbed solar value) along the sunspot axis for the same instants of time as shown in Fig. 2 (note the different depth range). Above the Wilson depression, the energy transport is taken over by the radiative flux (not shown here). The convective transport affects progressively deeper layers in order to supply the (almost constant) surface energy flux density of about $22 \%$ of the undisturbed solar value. The corresponding downward extension of the superadiabatic stratification leads to a growth of the region that is stabilized against disconnection through the resulting increase of the magnetic field (cf. Fig. 2.

that reflects the not quite realistic initial condition. As time progresses, the depth gradient of the magnetic field strength flattens and a local minimum develops, which becomes deeper and moves downward while, at the same time, there is a slow increase of the field strength in the upper layers. Both effects are connected to the development of a superadiabatic stratification in the sunspot model, which results from the growth of the region with convective energy transport. This is illustrated in Fig. 3, which shows depth profiles of the convective energy flux density (normalized by the undisturbed solar value) for the same instants as the magnetic field profiles in Fig. 2 (note the different depth scale). The superadiabatic stratification in the region with significant convective energy flux leads to a decrease of the pressure scale height and an associated increase of the field strength and thus prevents the field from becoming weak in the upper layers. As the superadiabatic region grows downward, the position of the field strength minimum also is shifted downward until the field strength eventually falls below the local equipartition level at a depth of about $4.7 \mathrm{Mm}$. The field strength at the bottom of the model has then reached about $10 \mathrm{kG}$, which is consistent with the results of thin flux tube simulations. The observable layers above the Wilson depression (optical depth unity, where the field strength remains around $2500 \mathrm{G}$ ) are largely unaffected by this whole development.

\section{Dependence on parameters}

The simulation run presented in the previous subsection yields values for the time scale and the depth of the disconnection that appear to be reasonable in view of what is indicated by observations of solar magnetic regions. How do these results
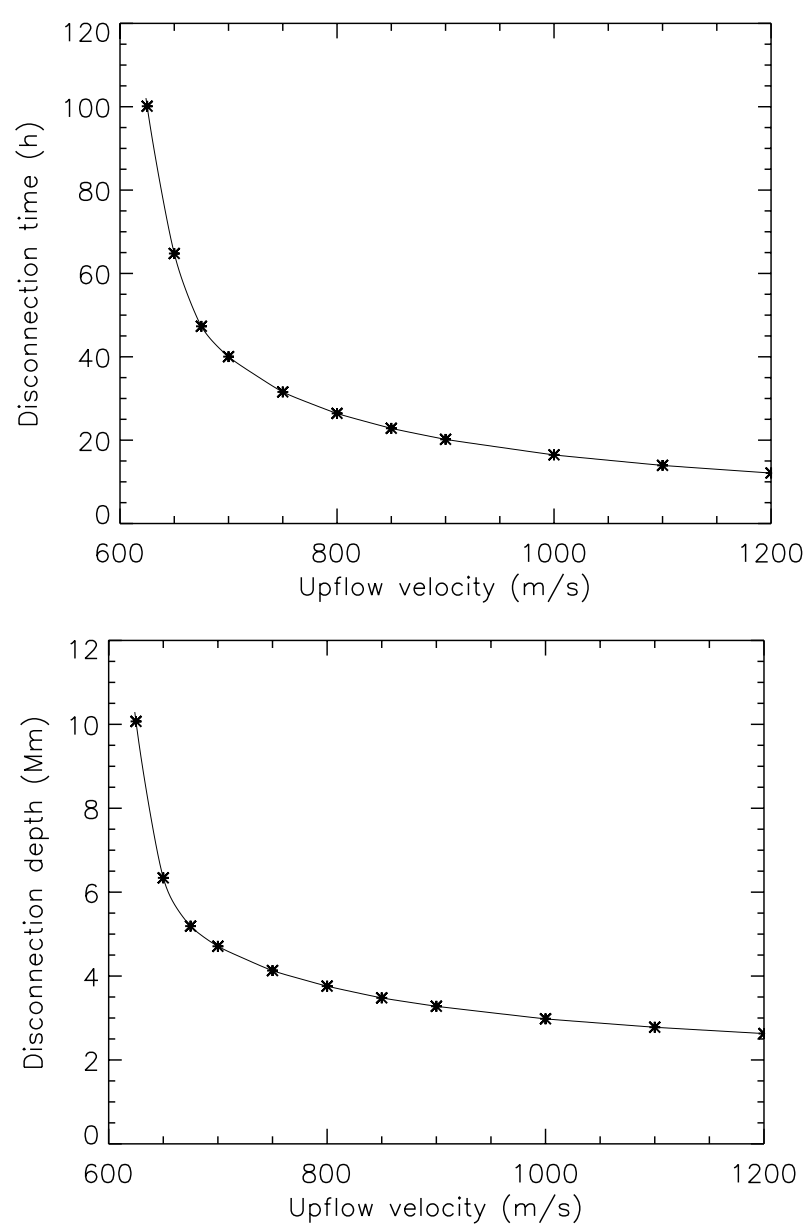

Fig. 4. Disconnection time (upper panel) and disconnection depth (lower panel) as functions of the initial upflow velocity at the lower boundary. The mass influx is kept constant in time in the individual simulation runs (indicated by asterisks). The connecting curves represent spline interpolations.

depend on the various parameters and assumptions entering the model?

The most important parameter of the model is the inflow velocity at the bottom, which determines the rate of pressure buildup at the base. For the case of constant mass flux through the bottom, Fig. 4 gives the disconnection time and depth (determined as time and depth for which the field strength first equals the external equipartition value) as functions of the initial inflow velocity, all other parameters being the same as those of the reference run discussed above. The plots show that that smaller values of the mass flux (smaller initial inflow velocity) lead to later and deeper disconnection. In the alternative case with constant inflow velocity (as opposed to constant mass influx), even much smaller inflow speeds of the order of $100 \mathrm{~m} \mathrm{~s}^{-1}$ lead to disconnection within few days and in a few Mm depth.

For high initial inflow speeds, the disconnection depth and time become largely independent of speed and reach values around $9 \mathrm{~h}$ and $2.3 \mathrm{Mm}$, respectively. This saturation results from the low entropy of the gas in the upper layers of the model due to the strong surface cooling, so that a strong field is always maintained in these layers. In fact, switching off the radiative 
losses invariably leads to a dramatic weakening of the magnetic field at the very top of the integration domain (depth zero).

We have varied a number of other parameters as well as initial and boundary conditions in order to evaluate their effects on the disconnection depth $\left(z_{\mathrm{d}}\right)$ and disconnection time $\left(t_{\mathrm{d}}\right)$. We find that the results are rather insensitive to the choice of temperature and magnetic field at the top $(z=0)$. The same is true for the (constant) value of the entropy in the initial stratification. In most cases we have taken the initial entropy equal to that of the external medium at the bottom of the model (12.5 Mm depth). Since the plasma in the flux tube originates from deeper layers zone, its entropy could be somewhat larger. However, since nearly all of the entropy drop through the convection zone takes place above $12.5 \mathrm{Mm}$, even taking the entropy value corresponding to the bottom of the convection zone has almost no effect on the results.

However, other parameters and conditions have a significant effect on the disconnection. It turns out that $z_{\mathrm{d}}$ and $t_{\mathrm{d}}$ decrease

- with decreasing depth of the lower boundary;

- with decreasing total magnetic flux;

- with increasing initial mass content;

- and also by keeping the inflow velocity (in contrast to the mass influx) constant in time.

Likewise, determining the magnetic field by replacing the similarity solution by the thin flux approximation (taking the limit $\Phi \rightarrow 0$ in Eq. (5)) leads to more shallow and earlier disconnection.

The quantitative results are combined in Fig. 5, which gives $z_{\mathrm{d}}$ as a function of $t_{\mathrm{d}}$ for a number of cases. Although the various simulation runs represent quite different conditions and assumptions, the results suggest a universal relationship between disconnection time and disconnection depth. The curve drawn in the figure is not a fit but gives, as a function of time, the depth at which the convective energy flux density equals $10 \%$ of the undisturbed solar value, about half of the emergent surface flux density. This curve therefore reflects the downward progression of the region of convective cooling as a result of the radiative losses at the surface (see Fig. 3).

The relationship between the disconnection depth and the downward progressing convective "cooling front" is in accordance with our interpretation of the disconnection depth in the case discussed in the previous subsection. Without surface cooling, the strongest weakening of the field would occur near to the top of the model. The cooling leads to the development of a superadiabatically stratified layer connected with a strengthening of the magnetic field in the upper part of the tube, which grows downward as the surface flux has to be supplied by the thermal energy stored in the deeper layers. This prevents the field in the upper layers from being weakened by the pressure buildup due to the inflow from below. The disconnection then takes place somewhere in the flank of the downward progressing convective flux profiles shown in Fig. 3, leading to the relation between $z_{\mathrm{d}}$ and $t_{\mathrm{d}}$ apparent from Fig. 5. In the absence of convective energy transport, the cool region would only extend down to the Wilson depression and the sunspot would be disconnected and disrupted immediately below that level, making

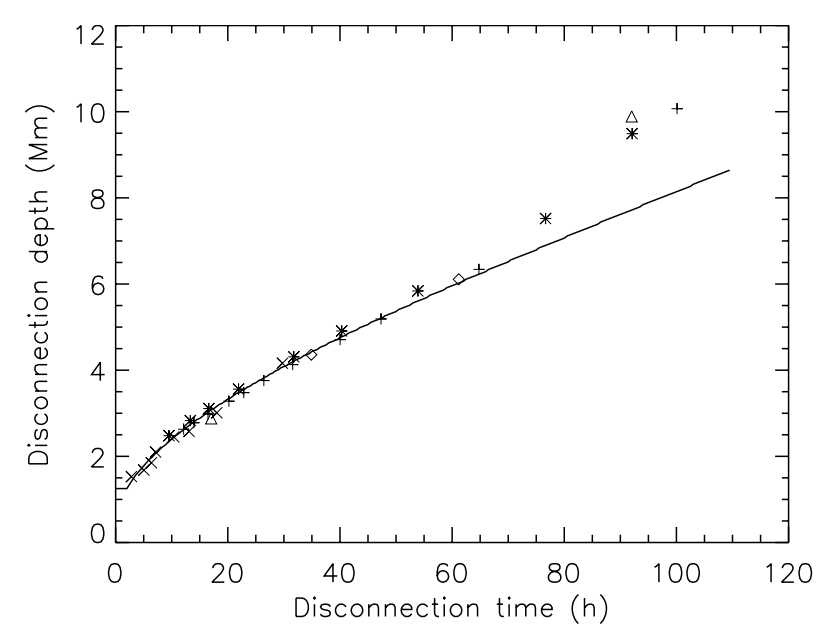

Fig. 5. Disconnection depth vs. disconnection time for various model runs. Plus signs: runs with constant mass influx (the same runs as in Fig. 4); asterisks: runs with constant inflow velocity in the range $75-400 \mathrm{~m} \mathrm{~s}^{-1}$; diamonds: runs with different values for the initial pressure at the top, changing the initial mass in the flux tube by about $\pm 10 \%$; triangles: runs with different values of the total magnetic flux $\left(10^{20} \mathrm{Mx}\right.$ and $3 \times 10^{21} \mathrm{Mx}$, respectively); crosses: runs for which the magnetic field was determined according to the pressure difference (approximation of thin flux tubes). The curve illustrates the downward progression of the zone with convective energy transport (cf. Fig. 3); it shows, as a function of time, the depth at which the convective energy flux density equals $10 \%$ of the undisturbed solar value, about half of the surface flux density of the model sunspot. Except for the largest disconnection depths (which may be affected by the proximity of the lower boundary of the model at $12.5 \mathrm{Mm}$ ), the points closely follow the curve, indicating that the disconnection depth is mainly determined by the downward progression of the convective cooling region.

it difficult to imagine that it could survive as a coherent entity thereafter.

This interpretation is further supported by runs with varied efficiency of convection, which affects the downward progression speed of the region of convective cooling. For the case shown in Figs. $1-3$, we have $\alpha=l / H_{\mathrm{p}}=0.3$, which gives a heat flux density ratio of $22 \%$. Larger (smaller) values of $\alpha$ lead to a faster (slower) downward extension of the convectively cooled region and to deeper (shallower) explosion: for $\alpha=0.4$ we find an explosion depth of about $6 \mathrm{Mm}$ and a flux of $30 \%$ of the undisturbed solar flux density, while a tube model with $\alpha=0.2$ explodes at a depth of $2.3 \mathrm{Mm}$ and shows only $14 \%$ of the undisturbed solar energy flux density. In both cases, the corresponding explosion depths and times are consistent with the downward propagation speed of the cooling flank.

\section{Discussion}

The results from our illustrative model indicate that dynamical disconnection of emerged magnetic flux is possible at a depth of less than about $10 \mathrm{Mm}$ and within a few days after emergence if upflow velocities as shown by simulations of thin flux tubes are considered (of the order of $\mathrm{a} \mathrm{km} \mathrm{s}^{-1}$ at $10 \mathrm{Mm}$ depth). However, we have to keep in mind that the assumption of a quasi-static state in the model forces us to use a too strong 
initial magnetic field in the deeper layers, so that the time evolution as shown here may not correctly represent what is actually happening below a sunspot. A more realistic time evolution of the disconnection process requires a full MHD simulation. On the other hand, the disconnected state represents a fully self-consistent solution with realistic surface properties and a hydrostatic stratification as indicated by observations (e.g., Beckers 1977). Our results show that a disconnected magneto-static sunspot model can be consistently constructed.

We have taken the equipartition field strength with respect to the convective velocities in the mixing-length model of the (non-magnetic) external medium as the limit for the onset of disconnection. This is only a rough indicator and the detailed disconnection process certainly is more complex. For instance, we have not considered the possible effect of fragmentation by the interchange (fluting) instability at the periphery of a flux tube bounded by a current sheet (Parker 1975). Meyer et al. (1977) have shown that buoyancy may stabilize a sunspot only down to a few Mm depth below the solar surface. Deeper down the destabilizing effect of the curvature of the surface bounding the flux tube dominates. In our case, the weakening of the field by the upflow leads to a hourglass-like shape of the sunspot, so that buoyancy effects in the lower part actually promote the instability. This could lead to fragmentation even before the equipartition limit has been reached.

The requirement for disconnection of emerged flux does not only apply to sunspots but covers all magnetic flux in bipolar regions. We suggest that the smaller flux tubes which form the plage and network regions are dynamically disconnected by the same process that affects the sunspots. In fact, using the thin flux tube approximation we find a shallow disconnection depth (of about $2 \mathrm{Mm}$ ) for such structures. This could be even smaller since a large part of the radiative energy loss of a small tube is provided by lateral radiative heating in the surface layers, so that the cooling of the subsurface layers proceeds more slowly than in sunspots, leading to a more shallow disconnection. It is well conceivable that the weakening of the field by the upflow in small flux fragments extends up to the surface, so that all initial connectivity is lost already during emergence or shortly thereafter. The formation of intense fields and the patterns of flux distribution in plages then results from the interaction with the local velocity fields at the solar surface (e.g., Vögler et al. 2005).

In which way does the fragmented flux below the disconnected surface flux evolve further? Since the flux fragments are passive with respect to convection, they will be deformed and stretched by the convective motions, probably keeping the field strength at about the equipartion value. However, this must not lead to a dynamical "re-connection" of the emerged part with its magnetic roots. We conjecture that this could be prevented by progressing fragmentation into ever thinner filaments, owing to various instabilities and dynamical processes (e.g., Schüssler 1984; Vishniac 1995). The fragmentation proceeds until the resistive length scale is reached, for which flux freezing is no longer valid and efficient exchange of mass and heat (by radiation) is provided (Schüssler 1987). The magnetic flux then has become truly passive and is kinematically transported by the motions of the plasma, providing no further possibility for restoring the dynamical link between the emerged flux and its the deep-lying parent flux tube. Such a transition to passive, weak field after the explosion of a flux tube has also been found in the numerical simulations of Rempel \& Schüssler (2001). Sustained outflow of buoyant plasma from the "stumps" of the disconnected parent flux tube and the horizontal motion driven by the curvature force until a narrow U-loop remains could also provide a mechanism for the removal of the parent flux tube from its storage region at the bottom of the convection zone. These processes can actually be observed in thin-tube simulations (Caligari et al., in preparation).

\section{Conclusion}

We have shown that, in the framework of a simple magnetostatic model, the dynamical disconnection of emerged magnetic flux from its magnetic roots due an upflow along the field is a rather robust feature, which occurs over a wide range of inflow velocities and within a reasonable interval of time after emergence. The disconnection depth lies between 2 and $6 \mathrm{Mm}$ for disconnection times less than 3 days, which is consistent with the observation that, shortly after the flux emergence phase, active regions do no longer reflect the dynamics of their magnetic "roots" deep in the convection zone. The disconnection depth is mainly determined by the extension of the radiatively and convectively cooled subsurface layer at the time of disconnection. Radiative cooling and downflow lead to a strengthening of the field in the upper layers and thus prevent a loss of equilibrium at the surface, which would lead to a complete destruction of the visible sunspot. Our results are consistent with findings from local helioseismology with regard to the subsurface structure of sunspots and active regions (e.g., Zhao et al. 2001; Couvidat et al. 2004).

Acknowledgements. This work has immensely benefited from numerous discussions and much exchange of ideas over the years with Fernando Moreno Insertis. Helpful comments by Yuhong Fan on a draft version of this paper are gratefully acknowledged. The National Center for Atmospheric Research (NCAR) is sponsored by the National Science Foundation.

\section{References}

Archontis, V., Moreno-Insertis, F., Galsgaard, K., Hood, A., \& O'Shea, E. 2004, A\&A, 426, 1047

Basu, S., Antia, H. M., \& Bogart, R. S. 2004, ApJ, 610, 1157

Baumann, I., Schmitt, D., Schüssler, M., \& Solanki, S. K. 2004, A\&A, 426, 1075

Beck, J. G. 2000, Sol. Phys., 191, 47

Beckers, J. M. 1977, ApJ, 213, 900

Caligari, P., Moreno-Insertis, F., \& Schüssler, M. 1995, ApJ, 441, 886

Choudhuri, A. R. 1992, in Sunspots. Theory and Observations, ed.

J. H. Thomas, \& N. O. Weiss (Kluwer, NATO ASI, C 375), 243

Couvidat, S., Birch, A. C., Kosovichev, A. G., \& Zhao, J. 2004, ApJ, 607, 554

Deinzer, W. 1965, ApJ, 141, 548

D'Silva, S., \& Choudhuri, A. R. 1993, A\&A, 272, 621

D’Silva, S., \& Howard, R. F. 1994, Sol. Phys., 151, 213 
Fan, Y. 2004, Living Reviews in Solar Physics, 1, 1 http://solarphysics.livingreviews.org

Fan, Y., \& Gibson, S. E. 2004, ApJ, 609, 1123

Fan, Y., Fisher, G. H., \& McClymont, A. N. 1994, ApJ, 436, 907

Ferriz-Mas, A., \& Schüssler, M. 1995, Geophys. Astrophys. Fluid Dyn., 81, 233

Fisher, G. H., Fan, Y., Longcope, D. W., Linton, M. G., \& Pevtsov, A. A. 2000, Sol. Phys., 192, 119

Hindman, B. W., Gizon, L., Duvall, T. L., Haber, D. A., \& Toomre, J. 2004, ApJ, 613, 1253

Javaraiah, J., \& Gokhale, M. H. 1997, A\&A, 327, 795

Kiefer, M., Grabowski, U., Mattig, W., \& Stix, M. 2000, A\&A, 355, 381

Kosovichev, A. G., Duvall, T. L. J., \& Scherrer, P. H. 2000, Sol. Phys., 192, 159

Magara, T. 2004, ApJ, 605, 480

McIntosh, P. S. 1981, in The Physics of Sunspots, ed. L. E. Cram, \& J. H. Thomas (Sunspot, NM, USA: Sacramento Peak Observatory, NSO), 7

Meyer, F., Schmidt, H. U., \& Weiss, N. O. 1977, MNRAS, 179, 741

Moreno-Insertis, F. 1997, Mem. Soc. Astron. It., 68, 429

Moreno-Insertis, F., Schüssler, M., \& Ferriz-Mas, A. 1992, A\&A, 264, 686

Moreno-Insertis, F., Caligari, P., \& Schüssler, M. 1995, ApJ, 452, 894

Parker, E. N. 1975, Sol. Phys., 40, 291

Parker, E. N. 1979, ApJ, 230, 905

Rempel, M., \& Schüssler, M. 2001, ApJ, 552, L171

Schlüter, A., \& Temesváry, S. 1958, in Electromagnetic Phenomena in Cosmical Physics, ed. B. Lehnert (Cambridge University Press), IAU Symp., 6, 263
Schrijver, C. J. 1987, A\&A, 180, 241

Schrijver, C. J. 2001, ApJ, 547, 475

Schrijver, C. J., \& Title, A. M. 1999, Sol. Phys., 188, 331

Schüssler, M. 1984, in The Hydromagnetics of the Sun (European Space Agency), ESA SP-220, 67

Schüssler, M. 1987, in The Internal Solar Angular Velocity, ASSL, 137, 303

Schüssler, M., \& Rempel, M. 2002, in From Solar Min to Max: Half a Solar Cycle with SOHO, ed. A. Wilson (ESTC, Noordwijk: ESA Publ. Div.), 499

Schüssler, M., Caligari, P., Ferriz-Mas, A., \& Moreno-Insertis, F. 1994, A\&A, 281, L69

Spruit, H. C. 1974, Sol. Phys., 34, 277

Spruit, H. C. 1981, in The Physics of Sunspots, ed. L. E. Cram, \& J. H. Thomas (Sunspot, NM, USA: Sacramento Peak Observatory, NSO), 98

Spruit, H. C., \& van Ballegooijen, A. A. 1982, A\&A, 106, 58

Strous, L. H., Scharmer, G., Tarbell, T. D., Title, A. M., \& Zwaan, C. 1996, A\&A, 306, 947

Thomas, J. H., \& Weiss, N. O. 1992, in Sunspots: Theory and Observations, ed. J. H. Thomas, \& N. O. Weiss (Kluwer, NATO ASI, C 375), 3

Tóth, L., \& Gerlei, O. 2004, Sol. Phys., 220, 43

Vögler, A., Shelyag, S., Schüssler, M., et al. 2005, A\&A, 429, 335 van Ballegooijen, A. A. 1982, A\&A, 106, 43

van Ballegooijen, A. A., Cartledge, N. P., \& Priest, E. R. 1998, ApJ, 501, 866

Vishniac, E. T. 1995, ApJ, 446, 724

Wang, Y.-M., Nash, A. G., \& Sheeley, N. R. 1989, ApJ, 347, 529

Zhao, J., Kosovichev, A. G., \& Duvall, T. L. 2001, ApJ, 557, 384 